\title{
Multi-scale cohesion force measurements for cemented granular materials
}

\author{
Abbas Farhat $^{1}$, Li-Hua Luu $^{1 *}$, Pierre Philippe ${ }^{1}$, and Pablo Cuéllar ${ }^{2}$ \\ ${ }^{1}$ RECOVER, INRAE Aix Marseille Univ, Aix-en-Provence, France \\ ${ }^{2}$ BAM, Federal Institute for Materials Research and Testing, Berlin, Germany
}

\begin{abstract}
.
We experimentally investigated cohesion of artificially bonded granular materials made of spherical glass beads cemented by solid paraffin bonds. By means of laboratory tests designed and carried out for investigation at different scales, we measured the tensile yield strength for solid bonds both at the inter-particle micro-scale and cemented samples at the meso-scale. A parametric study has been performed by varying some of the granular material properties (bead diameter, paraffin content as well as the dimension of the sample for the meso-scale tensile tests. We finally propose a discusion on: (i) the relationship between the microscopic and macroscopic cohesion forces relying on classical homogenisation laws ; (ii) the potential impact of size effects based on a simple phenomenological model.
\end{abstract}

\section{Introduction}

Characterization of soil cohesion is crucial for the understanding of geomechanical phenomena such as erosion, excavation, or sediment transport [1-4]. More generally, cohesive granular flows occur in many industrial processes ranging from agriculture to pharmaceutical. Recent works dealing with classical problems of flows through an orifice or down an inclined plane, showed how the presence of adhesive inter-particle forces reduces the "flowability" of powders and grains [5]. In a soil, cohesion can come from adhesion at direct contacts between two grains [6-8] or through a third body forming an adhesive bridge $[9,10]$. In the case studied here, we are dealing with artificial cemented soils containing solid bonds which are classically modelled by a cylindrical shape $[3,4,11]$. By measuring inter-particle adhesion using traction tests for both the local grain-scale and the sample meso-scale, we experimentally challenge the classical micro-macro relationship, as originally proposed by Rumpf [12].

\section{Experimental results}

\subsection{Samples' preparation}

The preparation method to produce our artificial cemented granular media has been previously presented in [3]. Briefly, spherical borosilicate glass beads with an uniform diameter $d$ and a density $\rho_{s}=2230 \mathrm{~kg} / \mathrm{m}^{3}$, are mixed with a given amount of hot liquid paraffin with a percentage by mass $x_{p}$. Note that the melting point of paraffin is around $40^{\circ} \mathrm{C}$ and that we paid particular attention to reach an homogeneous distribution without burning the mixture by using an optimal temperature of $90^{\circ} \mathrm{C}$. The cementation is

*e-mail: li-hua.luu@inrae.fr

A video is available at https://doi.org/10.48448/33p9-3x96 subsequently obtained through the solidification of paraffin by an over-night cooling at ambient temperature ${ }^{1}$. The top picture of Fig.1a shows an example of a solid bond between two particles. In this experimental study, we prepared samples of cohesive granular material with beads of diameters $d=1.4,3,4$, and $7 \mathrm{~mm}$, and paraffin contents equal to $x_{p}=0.2,0.5,0.7$, and $1 \%$.

\subsection{Micro-scale tensile tests}

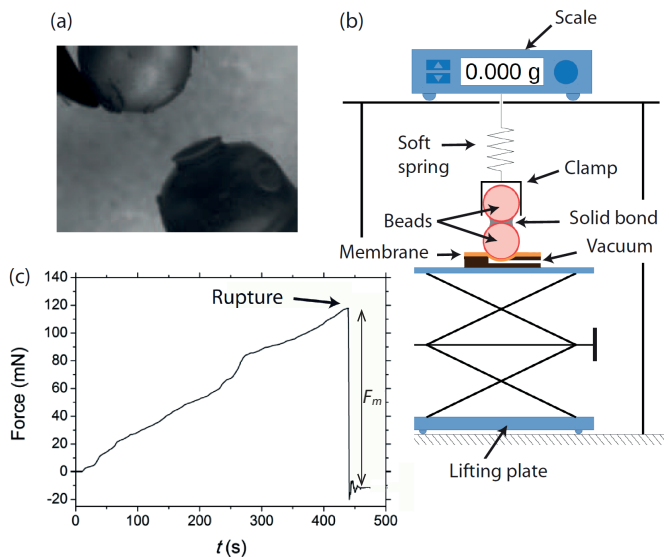

Figure 1. (a) Sketch of the micro-tensile test. (b) Pictures of bond breakage of a paraffin solid bridge between two glass beads ( $d=4 \mathrm{~mm}$ and $x_{p}=1 \%$ ). (c) Typical time evolution of the force measured during the test, giving the micro-tensile force $F_{m}$.

The setup of the mechanical test enabling the measurement of the tensile strength for an individual paraf-

\footnotetext{
${ }^{1}$ It was checked that 8 hours is enough to reach the ambient temperature inside of the sample for the larger specimen.
} 
fin solid bond is illustrated in Fig.1b. A pair of bonded beads is first carefully extracted from a sample of material and then set vertically in such a way that one of the beads is grabbed by a clamp and the other one is held fixed at the bottom plate either by strong glue or by suction using a membrane-vacuum system. From this initial stage, the bottom plate is slowly lowered until the solid bridge ultimately breaks. The upper clamp is connected to an inverted scale by means of a very soft spring that hence extends according to the lifting plate stroke generating at the bond scale a continuous increase of the tensile force with negligible deformation. At rupture, a mass increment $\Delta m$ is measured, giving rise to the micro-tensile force $F_{m}$, such as $F_{m}=\Delta m g$ with gravity $g=9.81 \mathrm{~m} / \mathrm{s}^{2}$ (Fig.1c). Notice that the use of paraffin induces an adhesive type of failure in which the paraffin bridge debonds from one of the grain surfaces (see Fig.1a bottom), instead of featuring a crack inside the bulk of the paraffin layer (cohesive failure).

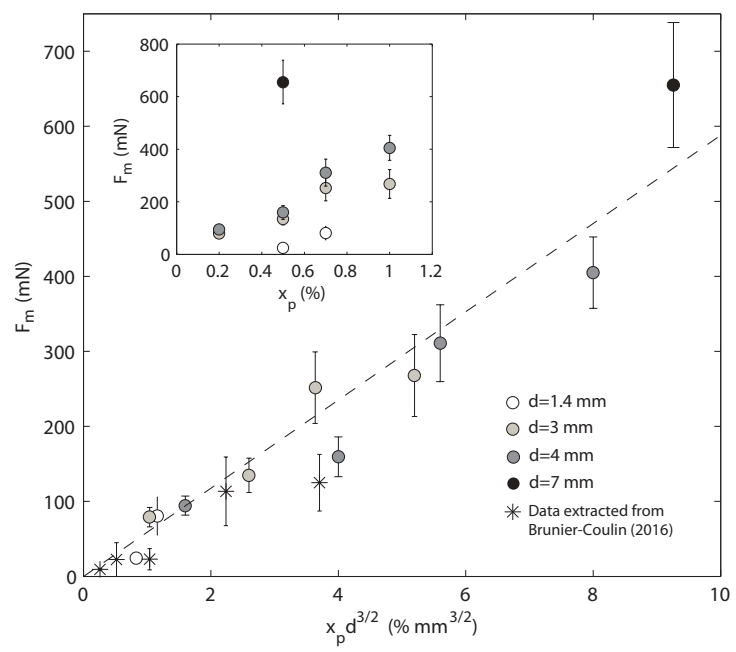

Figure 2. Micro-tensile force $F_{m}$ as a function of $x_{p} d^{3 / 2}$. The dashed line stands for $y=\alpha x$, with the fit parameter $\alpha=59 \pm 7$. Inset: $F_{m}$ as a function of $x_{p}$.

Figure 2 displays the particle-particle cohesion force $F_{m}$ for different bead diameters $d$ and paraffin concentrations $x_{p}$. Each point is obtained by averaging over at least ten measurements. Mainly due to intrinsic discrepancies, standard deviations on the mass measurements may exceed $50 \%$ whereas standard errors are around $20 \%$. We identified two limitations for the smallest particle size $d=1.4 \mathrm{~mm}$ : when $x_{p}=0.2 \%$, the bonds are so weak that the extraction of pairs of beads becomes a very challenging task; when $x_{p}=1 \%$, we observe the significant occurence of paraffin bonds involving more than two beads. The inset graph indicates that the higher $d$ and $x_{p}$, the higher the tensile strength $F_{m}$ required for bond rupture. The main graph shows that all measured values gather together satisfactorily when plotted as a function of $x_{p}$ multiplied by $d^{3 / 2}$. Similar experiments resulting from a previous work [4] are in a fairly agreement with this simple empirical relationship (star symbols).

\subsection{Macro-scale tensile tests}

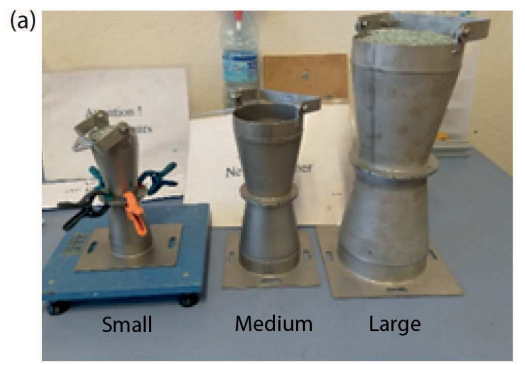

(b)

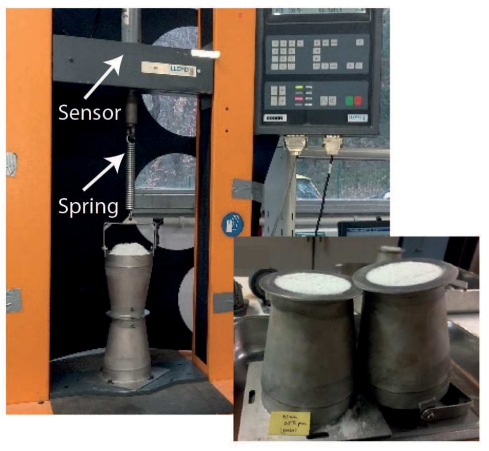

Figure 3. (a) The three sizes of the macro-scale tensile devices. (b) Picture of the traction bench. Inset: Typical failure surfaces after a test.

To measure the global cohesion force at the sample macro-scale, we performed traction tests using a cell constituted of two conical parts as pictured in Fig.3. In order to investigate potential boundary effects, we made three different sizes for the cell, varying the minimum cone frustrum diameter $(D=30,56$ and $79 \mathrm{~mm})$ while keeping a similar aspect ratio. Each cemented granular sample is directly prepared within the assembled cell. The lower part is fixed on the bottom of the traction bench while the upper part is attached via a spring to a force sensor which is fixed to a mobile platform. Then the mobile platform is slowly lifted upward at a constant velocity $(0.1 \mathrm{~mm} / \mathrm{min}$ for the small and medium devices, and $0.3 \mathrm{~mm} / \mathrm{min}$ for the large device) until separation of the two cones. The macro-tensile stress $\sigma_{M}$ is defined by the recorded force at rupture, denoted by $F_{M}$, divided by the failure section as follows:

$$
\sigma_{M}=\frac{F_{M}}{(\pi / 4) D^{2}} .
$$

Figure 4 represents typical variations of $\sigma_{M}$ as a function of the paraffin content $x_{p}$, depending on the bead diameter and on the size of the device, respectively denoted by small $(D=30 \mathrm{~mm})$, medium $(D=56 \mathrm{~mm})$ and large $(D=79 \mathrm{~mm})$. As expected, the macro-scale stress required for a failure increases with paraffin concentration, while it tends to slightly decrease when the particle size is enlarged (Fig.4a). However, we observe discrepancies in $\sigma_{M}$ measurements based on different cell sizes (at fixed $d$ and $x_{p}$ ), while a a clearer consistency would have been expected (Fig.4b). This is probably due to both some boundary effects inducing a lower solid fraction at the wall and the 
intrinsic inhomogeneity of bonds breakage events already evidenced by the micro-scale tests in section 2.2. We unified the dataset of measurements (i.e. with varying $d, x_{p}$ and $D$ ) in Figure 5. Relying on the power-law at microscale given in Fig. 2, $\sigma_{M}$ should roughly scale with $x_{p} d^{-1 / 2}$ as a first-order approximation. Despite substantial scatter, such a scaling is more or less consistent with our measurements as shown in Fig.5, especially in the inset where the data obtained for the three different sizes are averaged all together.
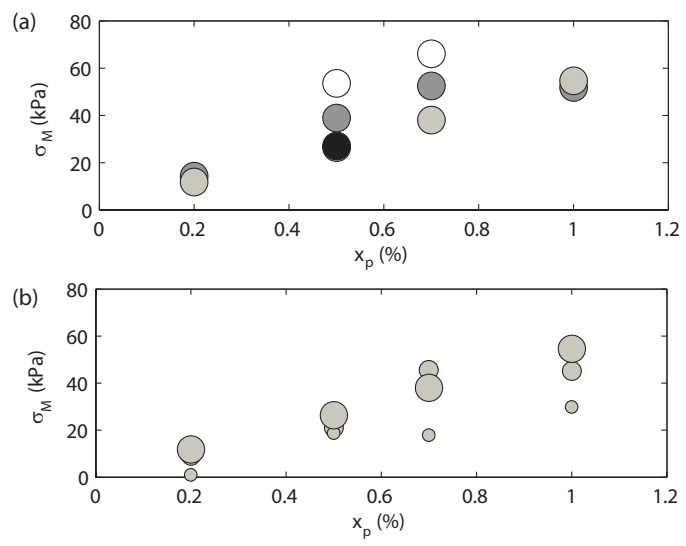

Figure 4. Macro-scale tensile stress $\sigma_{M}$ as a function of the paraffin content $x_{p}$. (a) Large device with $d=1.4 \mathrm{~mm}$ (white), $d=3 \mathrm{~mm}$ (grey), $d=4 \mathrm{~mm}$ (dark grey), and $d=7 \mathrm{~mm}$ (black). (b) Large, medium and small devices (symbol size) with $d=3 \mathrm{~mm}$.

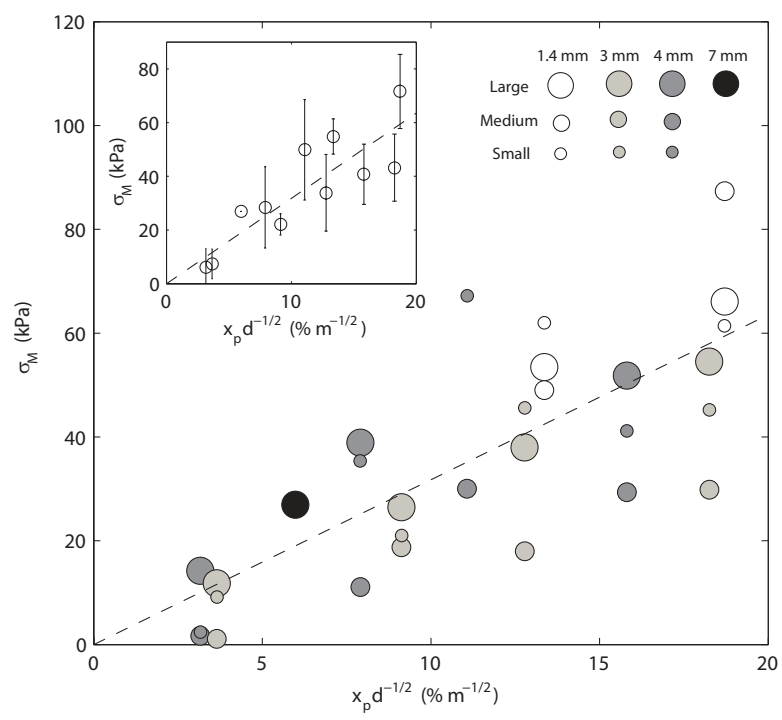

Figure 5. Macro-scale tensile stress $\sigma_{M}$ as a function of the paraffin content $x_{p}$ divided by $\sqrt{d}$. Inset: Averaged values. The dashed line stands for $y=\beta x$, with the fit parameter $\beta=3.2 \pm 0.4$.

\section{Discussion}

Now that we have separately measured the microscopic and the macroscopic yield tensile forces, we aim next to fully characterize the cohesion of our cemented granular material by relating these two scales. Since the pioneering work of Rumpf [12], different studies have developed several similar formulas that relate linearly the macroscopic cohesive stress to the inter-particle adhesion force [1315]. In Richefeu et al. [15], the authors propose considering the macroscopic stress as $\sigma_{M}=\frac{1}{2} n\left\langle f_{n} l\right\rangle$, where the symbol $\langle\ldots\rangle$ stands for averaging over all bonds in the control volume, $f_{n}$ is the normal component of the force, $l$ is the length of the branch vector, and $n$ is the density of bonds. The latter quantity reads $n=\frac{Z_{c} / 2}{V_{p} / \phi}$, where $Z_{c}$ is the coordination number, $\phi$ the solid volume fraction, and $V_{p}=\pi d^{3} / 6$ the particle volume. Assuming that $\left\langle f_{n} l\right\rangle=F_{m} d$, one gets:

$$
\sigma_{M}=\frac{3}{2} \frac{\phi Z_{c}}{\pi d^{2}} F_{m}
$$

This prediction is a factor $3 / 2$ higher than the initial Rumpf's law [12]. In the present study, we considered the variation in the coordination number. By injecting Eq.1 into Eq.2, the coordination number is given by:

$$
Z_{c}=\frac{8}{3 \phi} \frac{F_{M}}{F_{m}}\left(\frac{d}{D}\right)^{2} .
$$

Figure 6a represents $Z_{c}$ calculated from the micro and macro-scale traction data presented above, as a function of the ratio $D / d$ of the macro-device cone's diameter $D$ to the grain's diameter $d$. Noting the large scatter discussed previously, we obtain values ranging from 1 to 17 ! Note that Eq. 3 is a theoretical law assuming a uniform and isotropic sample, used as a basis for this analysis. As a perspective, particle-scale numerical modelling such as DEM should be considered to validate further this approach. There is in particular a noticeable decrease in $Z_{c}$ for the lowest paraffin content $x_{p}=0.2 \% \mathrm{~mm}$. This latter behavior is generally consistent with the numerical study by Richefeu et al. [15], where the coordination number of wet granular materal slighty increased with the water content. Figure $6 \mathrm{~b}$ provides a crude average of the previous $Z_{c}$ values in six successive ranges of $D / d$ whatever the other parameters (bead diameter, size of the macro-device, paraffin content), showing an almost constant value around $7 \pm 2$. If we consider the usual relationship $Z_{c}=\pi /(1-\phi)$ proposed in [13], we get $Z c=8.06$ for $\phi=0.61$ as estimated in our experiments. So, despite the large scatter in the data, a general agreement with classical approaches seems to emerge without any further calibration.

Finally, we consider to what extent the finite size of the samples could impact the coordination number. To this end, we built a model based on simple considerations to take into account the influence of the lateral wall. Let's first define the total number of grains in the rupture section as $N=\phi \frac{\pi D^{2}}{\pi d^{2}}$, and the number of grains in contact with the lateral wall as $N_{w}=\phi \frac{\pi D}{d}$. Denoting the bulk coordination 
number by $Z_{c}^{*}$, we propose to write the mean coordination number $Z_{c}$ as follows:

$$
Z_{c}=\frac{N-N_{w}}{N} Z_{c}^{*}+\frac{N_{w}}{N}\left(1+\frac{Z_{c}^{*}}{2}\right) .
$$

In this expression, we considered that: (i) a grain with no contact with the wall has $Z_{c}^{*}$ contacts with other grains ; (ii) a grain in contact with the wall has only one contact (the one with the wall) in the half space towards the wall, reflecting a lower solid fraction at the boundary, and $Z_{c}^{*} / 2$ contacts in the opposite half space. The following alternative expression can also be found:

$$
Z_{c}=Z_{c}^{*}\left[1-\pi \frac{d}{D}\left(\frac{1}{2}-\frac{1}{Z_{c}^{*}}\right)\right] .
$$

By using $Z_{c}^{*}=8.06$ as estimated above, the present phenomenological model predicts a significant boundary effect only for $D / d<10$ (dotted line in Fig.6b), which is indeed out of our experimental range. This suggests that no relevant finite size effect is expected here.
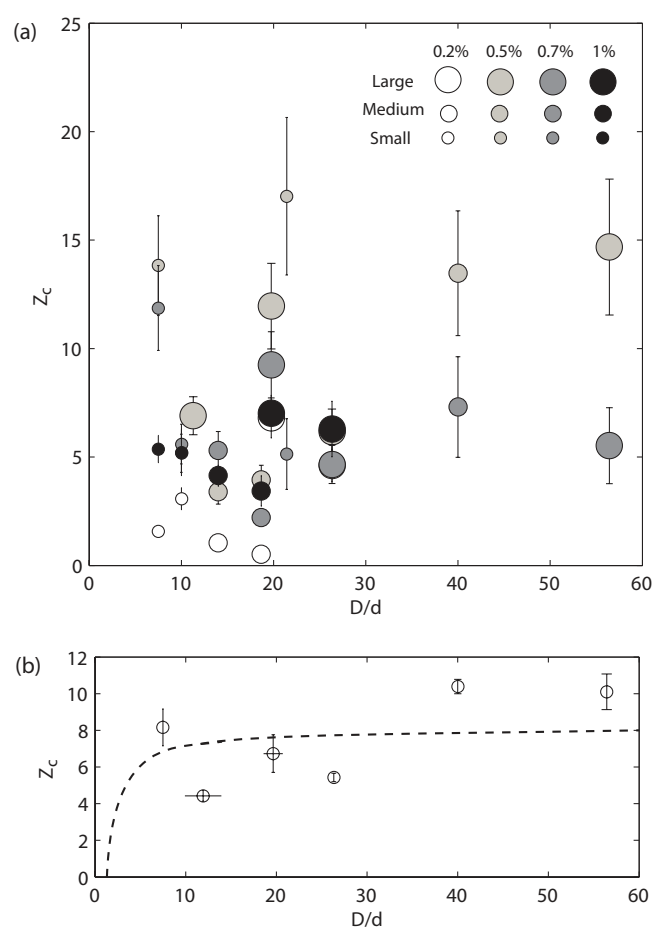

Figure 6. (a) Coordination number $Z_{c}$ as a function of $D / d$. The caption indicates the meso-scale devices' size and the paraffin content. (b) Averaged values of $Z_{c}$ as a function of $D / d$. The dotted line stands for the theoretical prediction from Eq.5.

\section{Conclusion}

The present contribution focused on the experimental characterization of artificial cemented granular materials. From micro-tensile tests, we measured a particle-particle force $F_{m}$ that increases with both the paraffin content $x_{p}$ and the bead diameter $d$. From macro-tensile tests, we also observe an increase of the tensile stress $\sigma_{M}$ with the amount of paraffin. However, the expected consistency of $\sigma_{M}$ through different cell sizes is not evident here. In the scope of our study, we empirically found that $F_{m}$ is proportional to $x_{p} d^{3 / 2}$, and that consequently $\sigma_{M}$ roughly scales with $x_{p} d^{-1 / 2}$. In a final discussion, we tested our data with a classical relationship between $F_{m}$ and $\sigma_{M}$ derived by [15]. The resulting coordination number $Z_{c}$ thus predicted fairly agrees with the expected value around 8 . Finally, a simple model allowed to confirm that finite size effects do not significantly impact our experimental findings. This step of characterization for our model soil now opens the possibility to consider a range of problems relevant to cohesive granular matter, as for instance erosion of soil subject to different types of flow configurations.

This work was supported by the ANR COMET project, grant ANR-2018-CE92-0007 of the French Agence Nationale de la Recherche.

\section{References}

[1] P. Philippe, P. Cuéllar, F. Brunier-Coulin, L.H. Luu, N. Benahmed, S. Bonelli, J.Y. Delenne, EPJ Web Conf. 140, 08014 (2017)

[2] L.H. Luu, G. Noury, Z. Benseghier, P. Philippe, Eng. Geol. 260, 105249 (2019)

[3] F. Brunier-Coulin, P. Cuéllar, P. Philippe, Phys. Rev. Fluids 5, 034308 (2020)

[4] F. Brunier-Coulin, Etude des mécanismes élémentaires de l'érosion d'un sol cohésif (Doctoral disserta- tion, Aix-Marseille, 2016)

[5] J. Fan, L.H. Luu, G. Noury, G., P. Philippe, Granul. Matter 22, 1-15 (2020)

[6] S. Mandal, M. Nicolas, O. Pouliquen, Proceedings of the National Academy of Sciences 117, 8366-8373 (2020)

[7] K. Kendall, J. Phys. D 4, 1186 (1971)

[8] M. Abdel-Ghani, J.G. Petrie, J.P.K. Seville, R. Clift, M.J. Adams, Powder Technol. 65, 113-123 (1991)

[9] A. Hemmerle, M. Schröter, L. Goehring, Nature Sci. Rep. 6, 1-11 (2016)

[10] R. Ge, M. Ghadiri, T. Bonakdar, Z. Zhou, I. Larson, K. Hapgood, Powder Technol. 340, 299-310 (2018)

[11] S. Antonyuk, M. Khanal, J. Tomas, S. Heinrich, Mörl, L., Chem. Eng. Process. 45, 838-856 (2006)

[12] H. Rumpf, Chem. Ing. Tech. 30, 144-158 (1958)

[13] P. Pierrat, H.S. Caram, Powder Technol. 91, 83-93 (1997)

[14] T. Gröger, U. Tüzün, D.M. Heyes, Powder Technol. 133, 203-215 (2003)

[15] V. Richefeu, M.S. El Youssoufi, F. Radjai, Phys. Rev. E 73, 051304 (2006) 